\title{
Institutional distrust among gay, bisexual, and other men who have sex with men as a barrier to accessing pre-exposure prophylaxis (PrEP)
}

\author{
Meghan Peterson ${ }^{\mathrm{a}, \mathrm{b}}$, Kathryn Nowotny ${ }^{c}$, Emily Dauria ${ }^{d}$, Trisha Arnold ${ }^{\mathrm{e}}$ and Lauren Brinkley-Rubinstein ${ }^{\mathrm{f}, \mathrm{g}}$ \\ ${ }^{\mathrm{a}} \mathrm{S}$ chool of Public Health, Brown University, Providence, RI, USA; ${ }^{\mathrm{b} C e n t e r}$ for Prisoner Health and Human Rights, Miriam Hospital, Providence, RI,

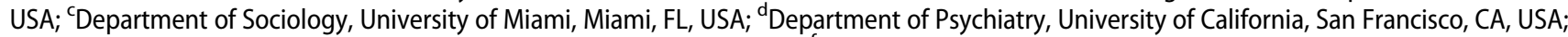 \\ 'Department of Psychology, Rhode Island Hospital, Providence, RI, USA; 'Department of Social Medicine, University of North Carolina, Chapel \\ Hill, NC, USA; ${ }^{9}$ Center for Health Equity Research, University of North Carolina, Chapel Hill, NC, USA
}

\begin{abstract}
Populations at highest risk for acquiring HIV are more likely to pass through criminal justice (CJ) settings, and CJ-involved individuals are often at the intersection of multiple overlapping risk factors. The present study explored interest in, knowledge of, and barriers to PrEP uptake among gay, bisexual, and other men who have sex with men involved in the criminal justice system. Using semi-structured interviews, 26 participants who identified as MSM were asked about PrEP knowledge and interest, HIV risk, and incarceration experience. One theme that emerged across interviews was how institutional distrust in CJ settings may instill lack of trust in medical care after perceived mistreatment. Participants explained how lack of privacy fostered feelings that medical care was not confidential, care received was tied to status as an incarcerated person, and feelings of dehumanization led to distrust. Findings explore how distrust may hinder PrEP uptake and other HIV prevention efforts in CJ settings as well as after release. They highlight the need for greater privacy efforts and cultural humility, and explore how medical settings may function as spaces for people who are incarcerated to disclose HIV risk status. Few studies to our knowledge have examined the role of institutional distrust on men who have sex with men (MSM) in the context of pre-exposure prophylaxis (PrEP) interventions. The present study has implications for creating best practices to structure HIV prevention interventions in $\mathrm{CJ}$ settings.
\end{abstract}

ARTICLE HISTORY

Received 3 February 2018

Accepted 29 August 2018

KEYWORDS

HIV/AIDS; pre-exposure prophylaxis (PrEP): incarceration; qualitative research

\section{Introduction}

HIV rates are three times higher among CJ (criminal justice) involved populations (Maruschak, 2012; Spaulding et al., 2009). People at risk for acquiring HIV are disproportionally likely to be incarcerated, including sexual minorities such as men who have sex with men (MSM) (Adimora, Schoenbach, \& Doherty, 2006; BrinkleyRubinstein et al., 2016; Meyer et al., 2017). During community re-entry individuals often engage in HIV risk behaviors, including condomless sex and sexual concurrency (i.e., overlapping sexual partnerships), making recently incarcerated individuals more vulnerable to HIV (Brewer et al., 2014; Tsui, Leonard, Lenoir, \& Ellen, 2008).

Given that incarcerated MSM are often at the intersection of many risk factors, HIV prevention interventions are needed for this subpopulation. One possible intervention for MSM with current or recent CJ involvement is pre-exposure prophylaxis (PrEP), a biomedical intervention that has shown efficacy in reducing HIV acquisition (Baeten et al., 2012; Grant et al., 2010;
Thigpen et al., 2012). Although initiation of PrEP has increased in the US since 2012, gaps may hinder PrEP uptake among at risk populations (Cáceres, Borquez, Klausner, Baggaley, \& Beyrer, 2016). Recent studies have demonstrated that PrEP knowledge among incarcerated individuals is low, but that interest is high once they learn more (Brinkley-Rubinstein et al., 2018; Dauria et al., 2017). However, multi-level (social and individual) barriers to PrEP are likely to be present and could negatively affect PrEP uptake and adherence (Arnold et al., 2017).

One possible barrier to PrEP uptake by MSM with CJ involvement is institutional distrust, or lack of trust in the CJ system and, subsequently, community-based healthcare systems. Sexual minorities and CJ-involved individuals have high levels of institutional distrust (Brayne, 2014; Puglisi, Calderon, \& Wang, 2017; Underhill et al., 2015). MSM involved in the CJ system are potentially at a "double disadvantage" given that trust in providers and medical structures can impact health outcomes (Armstrong et al., 2006; Lee \& Lin, 2009; 
Thom, Ribisl, Stewart, \& Luke, 1999). Fostering trust in medical settings can improve patient outcomes by increasing likelihood of receiving treatment and medication adherence (Lee \& Lin, 2009).

Exposure to negative treatment can create long-term aversions to care. Brayne (2014) documented how individuals with varying levels of $\mathrm{CJ}$ involvement develop distrust in societal institutions. The consequences are engagement in "system avoidance" wherein CJ involved persons avoid surveilling institutions that keep formal records such as hospitals. CJ involved individuals may have lower utilization of health services despite unmet need (Goffman, 2009). Formerly incarcerated individuals have specific health needs surrounding the transition to community-based resources, but often receive care from community providers who may not understand the extent to which past mistreatment, especially during incarceration, may influence current distrust (Puglisi et al., 2017).

In the current study, we describe the experience of institutional distrust among MSM $(n=26)$ who are currently incarcerated and the impact institutional trust may have on motivation for PrEP uptake both postrelease and in CJ settings.

\section{Methods}

The current study was conducted at the Rhode Island Department of Corrections (RIDOC) in Cranston, Rhode Island. The RIDOC is a statewide prison and jail that houses all incarcerated individuals in the state. Approximately 15,000 men cycle through the RIDOC each year (Rhode Island Department of Corrections, 2011), and the prevalence of HIV is 3\% (Rhode Island Department of Corrections, 2011).

Information related to sexual orientation is collected during medical intake, which occurs in the first $48 \mathrm{~h}$ after incarceration. We received a waiver of documentation of consent, so participants only gave verbal consent before the interview. Inclusion criteria for this study included self-report of: being gay, bisexual or a man who has sex with men; $\geq 18$; and being able to speak English. Interviews lasted 45-60 $\mathrm{min}$ and were conducted by three trained qualitative researchers (all female). Participants were asked questions related to HIV risk, PrEP knowledge and interest, barriers to PrEP uptake and adherence, and experience disclosing sexual orientation/identity. Interviewers provided a basic overview of PrEP to participants at the beginning of the interview and did not ask about PrEP knowledge before the interview began. Interviews were conducted in a private room without correctional officers (COs) present. Interviews were digitally recorded and transcribed. All participants received $\$ 30$ that was deposited into their commissary account. The study was approved by the institutional review board at the Miriam Hospital and RIDOC.

A general inductive approach was used to analyze data. Data were formulated into themes and categories in line with the research questions and objectives (Thomas, 2006). Two coders read through transcriptions looking for recurrent themes and patterns. Subsequently, each theme was given a code, and codes were compiled in a codebook. Quality checks were conducted on $20 \%$ of all transcripts for thematic agreement. Discrepancies in interpretation were resolved before final coding commenced.

\section{Results}

A total of 26 incarcerated MSM at the RIDOC were interviewed. Sixteen were White, 8 were Black, and 2 were Hispanic. Participants ranged in age from 23 to 57 and the average age was 38 . Across all interviews, participants described how institutional distrust within CJ medical systems hinders access to healthcare and, specifically, PrEP implementation, uptake and adherence. Below we present three major themes that relate to institutional distrust including: lack or privacy, lack of autonomy, and feelings of dehumanization. We describe how medical spaces in CJ facilities were sometimes considered safe spaces where institutional trust could be fostered.

Many participants discussed how lack of privacy in the CJ setting may instill fear that medical care is not confidential. Participants identified incidents in which proliferation of gossip - from both staff and incarcerated individuals - could prevent disclosure of PrEP status. Some participants mentioned a reluctance to disclose sexual identity/orientation to staff and other people who were incarcerated despite having disclosed at intake.

Several participants identified the role of COs in fostering an atmosphere devoid of privacy. While discussing sexual orientation, one participant commented:

Like I know COs listening when people are talking, so having like casual conversations or, it's, sometimes it's hard, the people who are [...] not totally honest with themselves they don't talk about it unless they're in their safe place wherever they go to, to play with other people. [participate in sexual activity]

Lack of privacy inherent to the facility therefore impeded willingness to disclose sexual orientation/identity. Another participant perceived that COs were knowledgeable of all interpersonal relationships, noting that: 
"[There's] nothing going on in this building that the cops don't know."

Some participants expounded on fear of physical violence, explaining that they felt disclosure and the inevitable spread of that information could compromise their safety. One participant commented that he initially hesitated to disclose to medical staff because he feared that other individuals might find out and threaten his wellbeing. He explained:

I was hesitant, like I said at first, I don't know why I was hesitant, probably just because you don't want people to find that out outside of that meeting, and like you don't want inmates to find that out because then all of a sudden if your identity is [revealed] people can make fun of you very easily in here [...] and who knows it could even spark people to, to want to physically get involved with you. Not physically in a good way, but to hurt you, you know.

This commentary highlighted ways in which perceived spread of information influenced care-seeking. When asked about barriers to beginning PrEP while incarcerated, one participant explained that others would have questions about his new medication regimen: "They always ask cause everyone is nosy here, $[\ldots]$ even the guards they all ask, like you know." He implied that while he would seek treatment, gossip could dissuade MSM who fear stigma. Ultimately, participants did not trust that information would remain private, hindering potential PrEP uptake and adherence.

Another theme that arose was a lack of autonomy surrounding medical decisions based on status as an incarcerated person. Participants felt they could not exert authority over medical decisions. Participants cited long lines to receive medications, delays in treatment, and feelings that they were not taken seriously when they expressed preferences surrounding medical treatment. Negative treatment and inability to make decisions surrounding health care led to many participants implying that they did not trust staff to provide them with appropriate care.

One participant noted that he stopped taking his medication because he did not have control over its administration: "I was on my psych meds. But I haven't been taking them anymore [...] Yeah, I wasn't able to. [...] Well [because] I didn't like it. Cause every morning you have to stay in the line ..." The participant reported that long lines and inadequate attention prevented him from taking medications that he had previously been prescribed.

Another participant questioned when he would receive his psychiatric medications despite feeling that they were important to his well-being:

They ordered them and they've been telling me that they've been ordered and that they should be [...] they should have it today, and I still have not been exposed to my psychiatric meds and those are very important to me because that's what keeps me at a level playing field. It's like I have really bad anxiety, and I have really bad like PTSD [...]

The participant felt disempowered in accessing his medications. He noted that he did not trust medical staff to obtain them based on being told conflicting information on when his medications would arrive. Many participants similarly explained how while they received medical care, they had little autonomy over decision-making. The lack of autonomy fostered feelings of distrust toward the system and providers at the RIDOC.

Some participants stated that when they received medical care they were dehumanized based on their status as an incarcerated person. They experienced incarceration-related stigma (Rose \& Clear, 2003; Schnittker \& John, 2007) and felt providers didn't think they "deserved" treatment. Participants implied that these experiences were disempowering and led to medical distrust.

While discussing feasibility of providing PrEP in a correctional setting, one participant explained: "[You] know a lot of people have different opinions about that, how much decision making and choice making a lot of us [people who are incarcerated] 'deserve' to have." The participant did not trust the system to provide adequate care based on his status as an incarcerated person.

Another participant expressed that he felt dehumanized by medical staff based on the treatment he received. He noted:

There's like plenty of people in here [staff], you know what I mean like they don't care about you, like they don't know, [...] his daughter might go to jail, they might pass through the same process that I'm passing right now, you know what I mean.

He felt that the level of care he received was influenced by the staffs perception of him as a person who was incarcerated and acknowledged that he felt dehumanized by staff s inability to imagine experiencing incarceration themselves.

Participants identified interactions with COs in which they felt that their medical care was trivialized. One participant explained:

I think that the guards here don't really take certain things seriously. You hear them cracking jokes about medline [a line where people receive their medications], about giving out meds that aren't really needed. I'd worry on the administrative end that [PrEP] wouldn't be taken seriously enough as something that was actually needed [...] Well the last time I was in medline and I overheard a guard making a comment about how the medline was so long that everybody was here 
taking drugs they don't actually need, and they're wasting money in the system for getting you know drugs.

Despite identifying many causes of institutional distrust, some participants expressed that they felt that medical spaces were more confidential than other spaces in CJ settings and medical staff could be more trusted. One participant explained:

I think that a lot of inmates would probably be more comfortable [disclosing sexual orientation/identity] with a member of medical staff as opposed to somebody who's involved on the floor or would be, or over you, such as a correctional officer or a lieutenant. [...] I know a lot of inmates don't really trust correctional officers, and [...] not all of them should be trusted.

This emphasized highlighted that individuals tended to trust medical staff more because they did not have disciplinary powers.

While explaining their medical intake process, a participant noted:

It was kind of comfortable in the little nurse's office at intake cause it was just a nurse and I think it was a clinical psychologist, a psychiatrist or psychologist sitting here and a guard. So it, you felt safe because you know that the guards not going to say anything and, and the two medical personnel aren't going to say anything so, so it felt safe there.

While many participants feared that information they gave would not be private, some identified medical settings as unique spaces where they could comfortably relay personal information.

However, when asked about experience disclosing sexual identity/orientation, participants had mixed reactions to doing so in a medical setting. One participant noted that the presence of COs and physical surroundings made him reluctant to disclose his sexual orientation during medical intake:

The combination of having you know open air surround and you know the $\mathrm{CO}$ was sitting there as well, so it wasn't like it was just myself and the nurse. [...] It was $\mathrm{CO}$ as well was in there with, with me when I answered the question, so I just you know was a little leery.

The view by some who are incarcerated of medical facilities as a "safe spaces" has implications for identifying best spaces to access populations at risk for acquiring HIV and fostering trust.

\section{Discussion}

The current study explored attitudes about PrEP uptake and institutional distrust among incarcerated MSM, a uniquely hard to engage population who are poorly represented in literature and are often at the intersection of multiple markers of risk. Results highlighted how lack of privacy instilled beliefs that medical care was not fully confidential, stigma due to incarceration status exacerbated perceptions of lack of autonomy in medical decision making, and negative and stigmatizing interactions with CJ staff (both COs and medical staff) and proliferation of gossip led to feelings of dehumanization. Despite general distrust, some participants noted that medical spaces in correctional facilities were a safer space to disclose sensitive medical information. However, not all participants shared this view with some noting that the open environment and presence of COs in the medical unit prevented them from feeling comfortable.

While few studies have explored institutional distrust from the perspective of incarcerated MSM, our findings corroborate existing research on institutional distrust among MSM, who are often reluctant to access health services due to stigma related to sexual orientation/identity (Franks et al., 2017; Gillman et al., 2013; Maulsby et al., 2014). Furthermore, it has been well documented that incarceration is often a first point of medical care for individuals with chronic health conditions, but people who are incarcerated often receive low quality care that is not patient-centered (Brinkley-Rubinstein \& Turner, 2013; Hatton, Kleffel, \& Fisher, 2006; Puglisi et al., 2017).

A unique finding of this study is how interactions with correctional health systems, not just incarceration itself (Hall, Dugan, Zheng, \& Mishra, 2001; Lee \& Lin, 2009), may lead to dehumanization and lack of autonomy in medical decision making. Many participants felt they received substandard care during incarceration - a perception that may affect future help-seeking behavior (Thom, Hall, \& Pawlson, 2004) both in the community and CJ settings. Participants felt that they exerted little control over their care. These findings highlight the need for greater privacy measures in CJ settings (especially surrounding medical care), patient-centered care that takes into consideration patient priorities and preferences, and cultural humility training for $\mathrm{CJ}$ staff. Participant comments on medical spaces as being "safer" than other parts of the facility indicate that these are the best spaces to deploy future interventions designed to facilitate PrEP uptake and bolster institutional trust. However, interventions should consider how open settings and presence of COs may dissuade individuals from disclosing sensitive information needed to screen for PrEP clinical indication. Processes for PrEP screening and disclosure of sexual identity/orientation should also incorporate information relevant to reasons for collecting risk information to foster trust that sexual 
identity/orientation will be used for relevant reasons. Additionally, screening processes must emphasis that this information will remain confidential and used appropriately.

Finally, an important implication of our results is the need to address the impact of interactions with medical staff in CJ settings on future willingness to seek care post-release. This could translate into more robust discharge planning, linkage to health services, and incorporation of client-centered approaches to understanding the longitudinal effect of CJ-based experiences on community-based help seeking. In addition, community-based health care providers should have access to incarceration-related cultural competency training. While considerable interest and high risk among MSM who are incarcerated indicate that PrEP could serve as a novel and effective intervention strategy, interventions must carefully address barriers to access outlined in the current study.

The current study has several limitations. The RIDOC is a statewide prison and jail system, whereas most correctional facilities in other states are either a jail or prison, and a larger proportion of incarcerated individuals in RI are White. The uniqueness of the RIDOC makes generalizability of our findings limited. However, these findings are meant to provide a snapshot of the lived experience of incarcerated MSM in RI only. Inclusion in this study relied upon individuals' selfreport of being MSM. Other participants may have been unwilling to disclose their sexual identity/orientation status due to fear of stigma from CJ entities meaning that the sample of people who did disclose is biased. Finally, the focus of these interviews was not explicitly to explore trust. Future studies should more broadly investigate institutional distrust in CJ settings.

\section{Conclusion}

The results of the current study suggest that incarcerated MSM may distrust medical systems and providers for a variety of reasons. Future PrEP interventions efforts must consider the impact of institutional distrust on PrEP uptake and adherence. There is a need to address confidentiality, past experiences with CJ settings and their impact on community-based PrEP uptake, and general strategies (including centering interventions in medical spaces) to foster trust. Furthermore, our findings support the need for research exploring the impact of medical distrust in other settings on PrEP uptake.

\section{Disclosure statement}

No potential conflict of interest was reported by the authors.

\section{Funding}

The present study was funded by the National Institute on Drug Abuse (NIDA) [grant numbers R25DA037190 and R25DA035692].

\section{References}

Adimora, A. A., Schoenbach, V. J., \& Doherty, I. A. (2006). HIV and African Americans in the southern United States: Sexual networks and social context. Sexually Transmitted Diseases, 33(7), S39-S45.

Armstrong, K., Rose, A., Peters, N., Long, J. A., McMurphy, S. \& Shea, J. A. (2006). Distrust of the health care system and self-reported health in the United States. Journal of General Internal Medicine, 21(4), 292-297.

Arnold, T., Brinkley-Rubinstein, L., Chan, P. A., PerezBrumer, A., Bologna, E. S., Beauchamps, L., ... Caylà, J. A. (2017). Social, structural, behavioral and clinical factors influencing retention in pre-exposure prophylaxis (PrEP) care in Mississippi. PLoS ONE, 12(2), e0172354.

Baeten, J. M., Donnell, D., Ndase, P., Mugo, N. R., Campbell, J. D., Wangisi, J., ... Celum, C. (2012). Antiretroviral prophylaxis for HIV prevention in heterosexual men and women. New England Journal of Medicine, 367(5), 399-410.

Brayne, S. (2014). Surveillance and system avoidance. American Sociological Review, 79(3), 367-391.

Brewer, R. A., Magnus, M., Kuo, I., Wang, L., Liu, T. Y., \& Mayer, K. H. (2014). The high prevalence of incarceration history among Black men who have sex with men in the United States: Associations and implications. American Journal of Public Health, 104(3), 448-454.

Brinkley-Rubinstein, L., Parker, S., Gjelsvik, A., Mena, L., Chan, P. A., Harvey, J., ... Nunn, A. (2016). Condom use and incarceration among STI clinic attendees in the Deep South. BMC Public Health, 16(1), 974.

Brinkley-Rubinstein, L., Peterson, M., Arnold, T., Nunn, A. S., Beckwith, C. G., Gastonguay, B., ... Chan, P. A. (2018). Knowledge, interest, and barriers of pre-exposure prophylaxis uptake and adherence among gay, bisexual, and other men who have sex with men who are incarcerated. Under Review at AIDS and Behavior.

Brinkley-Rubinstein, L., \& Turner, W. L. (2013). Health impact of incarceration on HIV-positive African American males: A qualitative exploration. AIDS Patient Care and STDs, 27(8), 450-458.

Cáceres, C. F., Borquez, A., Klausner, J. D., Baggaley, R., \& Beyrer, C. (2016). Implementation of pre-exposure prophylaxis for human immunodeficiency virus infection: Progress and emerging issues in research and policy. Journal of the International AIDS Society, 19(7S6), 717-723.

Dauria, E. F., Tolou-Shams, M., Levine-Murray, A., Lipman, M., Comfort, M., \& Christopoulos, K. (2017, June 5). "We need this!": PrEP awareness and acceptability among women involved in the criminal justice system. Oral Presentation. 12th International Conference on HIV Treatment and Prevention Adherence, Miami, FL.

Franks, J., Hirsch-Moverman, Y., Loquere, A. S., Amico, K. R., Grant, R. M., Dye, B. J., ... Mannheimer, S. B. (2017). Sex, PrEP, and stigma: Experiences with HIV pre-exposure prophylaxis among New York city MSM participating in the HPTN 067/ADAPT study. AIDS and Behavior, 1, 1139-1149. 
Gillman, J., Davila, J., Sansgiry, S., Parkinson-Windross, D., Miertschin, N., Mitts, B., ... Giordano, T. P. (2013). The effect of conspiracy beliefs and trust on HIV diagnosis, linkage, and retention in young MSM with HIV. Journal of Health Care for the Poor and Underserved, 24(1), 36-45.

Goffman, A. (2009). On the run: Wanted men in a Philadelphia ghetto. American Sociological Review, 74(3), 339-357.

Grant, R. M., Lama, J. R., Anderson, P. L., McMahan, V., Liu, A. Y., Vargas, L., ... Glidden, D. V. (2010). Preexposure chemoprophylaxis for HIV prevention in men who have sex with men. New England Journal of Medicine, 363(27), 2587-2599.

Hall, M. A., Dugan, E., Zheng, B., \& Mishra, A. K. (2001). Trust in physicians and medical institutions: What is it, can it be measured, and does it matter? The Milbank Quarterly, 79(4), 613-639.

Hatton, D. C., Kleffel, D., \& Fisher, A. A. (2006). Prisoners' perspectives of health problems and healthcare in a US women's jail. Women \& Health, 44(1), 119-136.

Lee, Y. Y., \& Lin, J. L. (2009). The effects of trust in physician on self-efficacy, adherence and diabetes outcomes. Social Science \& Medicine, 68(6), 1060-1068.

Maruschak, L. M. (2012). HIV in prisons, 2001-2010. AIDS, 20 (25.10), 1-11.

Maulsby, C., Millett, G., Lindsey, K., Kelley, R., Johnson, K., Montoya, D., \& Holtgrave, D. (2014). HIV among black men who have sex with men (MSM) in the United States: A review of the literature. AIDS and Behavior, 18(1), 10-25.

Meyer, I. H., Flores, A. R., Stemple, L., Romero, A. P., Wilson, B. D., \& Herman, J. L. (2017). Incarceration rates and traits of sexual minorities in the United States: National inmate survey, 2011-2012. American Journal of Public Health, 107(2), 267-273.

Puglisi, L., Calderon, J. P., \& Wang, E. A. (2017). What does health justice look like for people returning from incarceration? The AMA Journal of Ethic, 19(9), 903-910.

Rhode Island Department of Corrections. (2011). Data research and planning. Providence, RI: RIDOC.
Rose, D. R., \& Clear, T. R. (2003). Incarceration, reentry, and social capital. Prisoners once removed: The impact of incarceration and reentry on children, families, and communities, 189-232.

Schnittker, J., \& John, A. (2007). Enduring stigma: The longterm effects of incarceration on health. Journal of Health and Social Behavior, 48(2), 115-130.

Spaulding, A. C., Seals, R. M., Page, M. J., Brzozowski, A. K., Rhodes, W., \& Hammett, T. M. (2009). HIV/AIDS among inmates of and releasees from US correctional facilities, 2006: Declining share of epidemic but persistent public health opportunity. PLoS ONE, 4(11), e7558.

Thigpen, M. C., Kebaabetswe, P. M., Paxton, L. A., Smith, D. K., Rose, C. E., Segolodi, T. M., ... Brooks, J. T. (2012). Antiretroviral preexposure prophylaxis for heterosexual HIV transmission in Botswana. New England Journal of Medicine, 367(5), 423-434.

Thom, D. H., Hall, M. A., \& Pawlson, L. G. (2004). Measuring patients' trust in physicians when assessing quality of care. Health Affairs, 23(4), 124-132.

Thom, D. H., Ribisl, K. M., Stewart, A. L., \& Luke, D. A. (1999). Further validation and reliability testing of the trust in physician scale. Medical Care, 37(5), 510-517.

Thomas, D. R. (2006). A general inductive approach for analyzing qualitative evaluation data. American Journal of Evaluation, 27(2), 237-246.

Tsui, E. K., Leonard, L., Lenoir, C., \& Ellen, J. M. (2008). Poverty and sexual concurrency: A case study of STI risk. Journal of Health Care for the Poor and Underserved, 19 (3), 758-777.

Underhill, K., Morrow, K. M., Colleran, C., Holcomb, R., Calabrese, S. K., Operario, D., ... Mayer, K. H. (2015). A qualitative study of medical mistrust, perceived discrimination, and risk behavior disclosure to clinicians by US male sex workers and other men who have sex with men: Implications for biomedical HIV prevention. Journal of Urban Health, 92(4), 667-686. 